\section{Pedicled lower lid-sharing flap for full-thickness reconstruction of the upper eyelid}

Department of Plastic Surgery, Changhai Hospital, Second Military Medical University, Shanghai, China

Correspondence: CY Xue, Department of Plastic Surgery, Changhai Hospital, Second Military Medical University, Shanghai 200433, China. Tel/Fax: + 862131161806. E-mail: xcyfun@sina.com ${ }^{1}$ These authors contributed equally to this study.

Received: 28 January 2014 Accepted in revised form: 26 June 2014 Published online: 8 August 2014

\begin{abstract}
Purpose To explore the clinical effect of the pedicled lower lid-sharing flap for fullthickness reconstruction of the upper eyelid. Methods From 2009 to 2013, 13 upper eyelids with meibomian gland carcinoma (13 patients, age range 52-78 years) were excised, and immediately reconstructed with a pedicled lower lid-sharing flap used for full-thickness upper eyelid defects (up to two-thirds of the eyelid width). Traditionally, the flap is divided after 3 to 4 weeks, and the recipient site closed directly.

Results During a 1-18-month follow-up period, no recurrence, lagophthalmos, hypertrophic scar, or bulky appearance was noted in any of the patients. Aesthetic results for the upper eyelid were obtained for all patients. Conclusions We conclude that the pedicled lower lid-sharing flap is a safe and reliable method for reconstruction of full-thickness upper eyelid defects. This procedure not only enables eyelid closure for eye protection, but also directly improves the aesthetic appearance of the face. After second-stage surgery, a stable eyelid margin and lashes with good blood supply and an acceptable cosmetic appearance with regard to symmetry of eyelid height, contour, tarsal show, and skin fold were achieved.

Eye (2014) 28, 1292-1296; doi:10.1038/eye.2014.183; published online 8 August 2014
\end{abstract}

\section{Introduction}

Eyelids are continuously visible and function in protecting eye sight. Full-thickness eyelid defects often lead to conjunctival irritation, keratopathy, conjunctival inflammation, corneal ulceration, and even blindness. The upper eyelid is located on the upper third of the face; perhaps no other area of the human body provides such a delicate interplay of anatomy, aesthetics, and function. In order to protect the underlying globe and vision, eyelids require restoration of both function and appearance following eyelid repair. Between August 2009 and December 2013, by adhering to the principles of 'like-for-like' when applying lower lid-sharing replacement and vascular supply, 13 patients with meibomian gland carcinoma of the upper eyelid underwent surgical resection and reconstruction. This technique allowed closure of full-thickness defects of greater than one-third of the eyelid width. Significant therapeutic effect and aesthetic results in the upper eyelid were obtained for all patients.

\section{Materials and methods}

Thirteen patients (eight men and five women, age range 52-78, median 68 years) underwent resection of meibomian gland carcinomas that resulted in upper eyelid full-thickness defects from 33 to $63 \%$ of the eyelid width. Patient demographic information, including gender, age, relative defect size, and other types of reconstruction, are summarized in Table 1.

\section{Operative technique}

All operations were performed under general anesthesia. A 5-mm incision was made along the carcinoma margin and the full-thickness upper eyelid was completely excised. Surgical specimens were immediately frozen and sent to the pathology laboratory for examination; only after the pathologist confirmed that there was no residual tumor in the specimen was the planned reconstruction undertaken. The pedicled lower lid full-thickness flap is triangular in design, with the horizontal length designed to be 
Table 1 Personal data for selected patients

\begin{tabular}{|c|c|c|c|c|c|c|c|c|}
\hline Patient no. & Age (years) & Gender & $\begin{array}{l}\text { Size of palpebral } \\
\text { fissure }(\mathrm{cm})\end{array}$ & $\begin{array}{c}\text { Size of } \\
\text { defect }(\mathrm{cm})\end{array}$ & $\begin{array}{c}\text { Size of } \\
\text { defect }(\%)\end{array}$ & $\begin{array}{l}\text { Size of flap } \\
\quad(\mathrm{cm})\end{array}$ & $\begin{array}{l}\text { Size of flap } \\
(\%)\end{array}$ & Other type of reconstruction \\
\hline 1 & 67 & Male & 3.6 & 1.3 & 36 & 0.8 & 22 & \\
\hline 2 & 69 & Male & 3.5 & 2.2 & 63 & 1.3 & 37 & Lateral cantholysis and Z flap \\
\hline 3 & 78 & Male & 3.3 & 1.5 & 45 & 1.0 & 30 & \\
\hline 4 & 74 & Female & 3.7 & 1.6 & 43 & 1.1 & 30 & \\
\hline 5 & 68 & Male & 3.5 & 1.4 & 40 & 0.9 & 26 & \\
\hline 6 & 64 & Male & 3.5 & 2.2 & 63 & 1.3 & 37 & \\
\hline 7 & 59 & Female & 3.6 & 2.1 & 58 & 1.2 & 33 & Lateral cantholysis and Z flap \\
\hline 8 & 73 & Male & 3.3 & 1.6 & 48 & 1.0 & 30 & $\begin{array}{l}\text { Medial cantholysis plasty in } \\
\text { one-step procedure }\end{array}$ \\
\hline 9 & 52 & Male & 3.5 & 1.3 & 37 & 0.8 & 23 & \\
\hline 10 & 58 & Male & 3.6 & 1.2 & 33 & 0.7 & 19 & \\
\hline 11 & 75 & Female & 3.5 & 2.0 & 57 & 1.2 & 34 & Lateral cantholysis and Z flap \\
\hline 12 & 77 & Female & 3.5 & 1.5 & 43 & 0.9 & 26 & $\begin{array}{l}\text { Medial cantholysis plasty in one- } \\
\text { step procedure }\end{array}$ \\
\hline 13 & 60 & Female & 3.6 & 1.7 & 47 & 1.1 & 31 & \\
\hline
\end{tabular}

less than the width of the defect (one-third to one-fifth of the eyelid width), in order to ensure an equal width between the upper and lower eyelids after reconstruction. The flap was harvested from the lower eyelid and rotated based on the palpebral edge artery. The distal cut end of the flap was inset by suturing the conjunctiva, tarsal plate, orbicularis muscle, and skin to the medial edge of the defect. The lower eyelid defect was directly closed when possible; if it was difficult to directly close the defect, a lateral cantholysis was performed and myocutaneous advancement flap, such as a Z flap, was used, with debonding of the medial canthal tendon where necessary. The flap was typically divided 3-4 weeks after surgery, at the level of the newly reconstructed upper eyelid margin, and the recipient site closed directly.

\section{Results}

The wounds of all the patients healed primarily and the flaps survived without complications. During the 1-18month follow-up period, no recurrence, lagophthalmos, hypertrophic scar, or bulky appearance was noted in any of the 13 patients. This procedure not only enabled eyelid closure for eye protection, but also directly improved the aesthetics of the face. After the second-stage surgery, a stable eyelid margin, lashes, good blood supply, and an acceptable cosmetic appearance with regard to symmetry of eyelid height, contour, tarsal show, and skin fold, were achieved. All patients were pleased with the functional and cosmetic results (Figure 1).

\section{Discussion}

By far, the most common causes of full-thickness eyelid defects are tumor resection and trauma. Small defects can be closed directly. Although upper eyelid defects as large as $20 \%$ can be closed directly in young patients, increased laxity in older patients allows for direct closure of defects as large as $30 \%$. However, for larger defects, wound dehiscence and secondary mechanical ptosis are a concern when using this technique in upper eyelid repair. Hence other methods such as the anterior lamella with placement of an overlying skin graft, bridge flap (Cutler-Beard), temporal forehead flap (Fricke), semicircular rotational flap (Tenzel), and the horizontal V-Y myotarsocutaneous advancement flap are required. ${ }^{1,2}$

Eyelids are continuously visible and function in protecting sight. Upper eyelid defects are one of the most challenging areas in reconstruction because of the highly specialized anatomy, aesthetics, and function of the upper eyelid. Ideally, any reconstruction should reach the following goals: (1) the eyelid must be mobile, so it can wipe the cornea, refresh the tear film, and clear the visual axis; (2) achieve the three layers to constitute the upper eyelid, with each tissue performing its specific function (the mucosa to protect the underlying cornea, the tarsus to stabilize the eyelid, the orbicularis to enable eyelid closure, the loose preseptal skin to allow mobility, and the tight pretarsal skin and lid margin to prevent occlusion or irritation of the cornea by preseptal skin); (3) the eyelid must have a stable eyelid margin that prevents stratified squamous epithelium, lashes, or hairs from abrading the cornea; (4) as the upper eyelid forms one of the most visible features of the human face, there should be an acceptable cosmetic appearance in relationship to the other eye-specifically symmetry of eyelid height, contour, tarsal show, and skin fold. Owing to the highly specialized anatomy, aesthetics, and function of the upper eyelid, and despite the anterior lamella being 

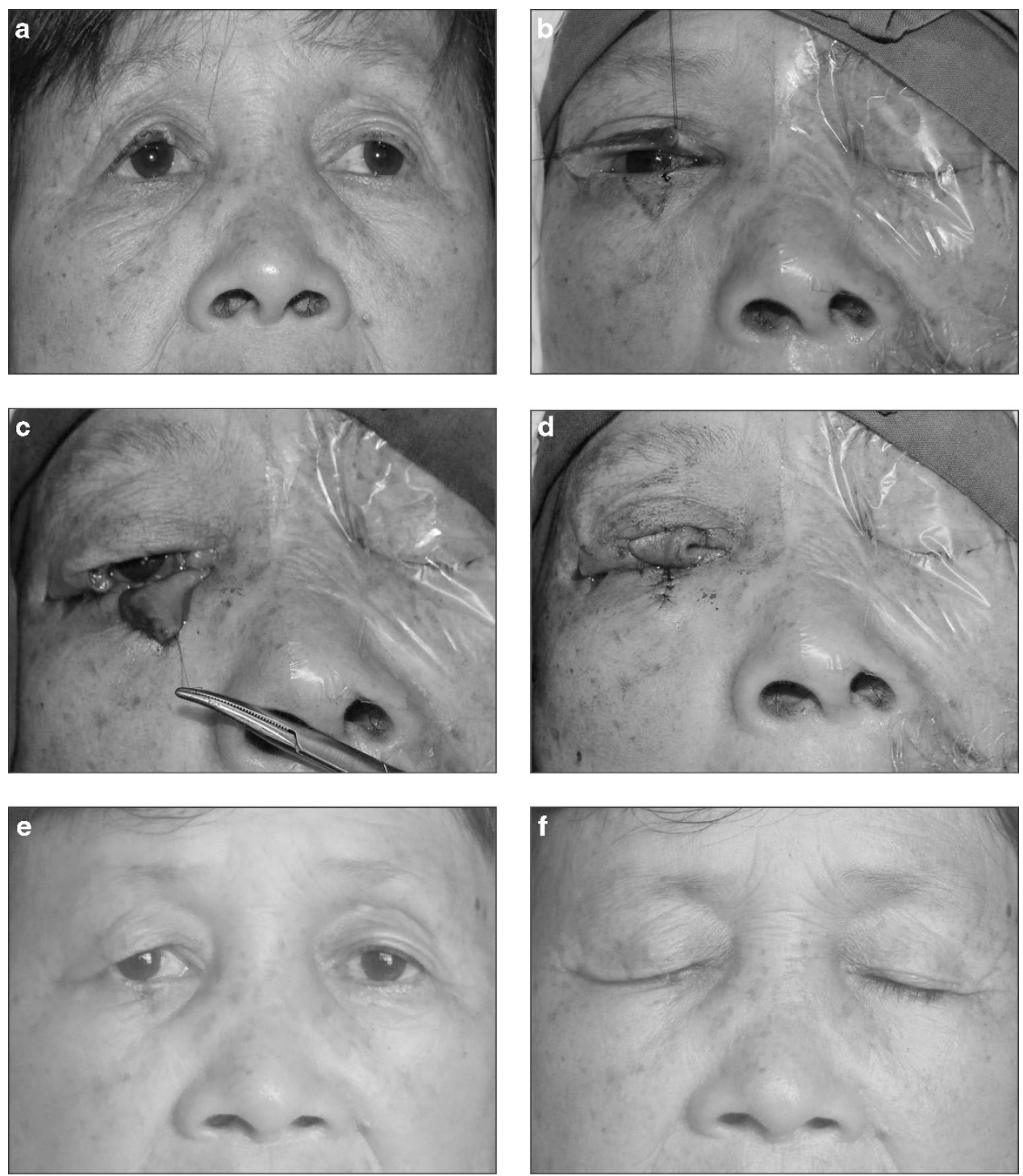

Figure 1 75-year-old female. (a) Preoperative view; (b) local resection creating a $2.0 \times 1.1 \mathrm{~cm}^{2}$ defect; (c) the flap is taken from the lower eyelid and incision of $\mathrm{Z}$ flap; (d) transfer of the flap to the upper eyelid defect; (e) secondary division of the pedicle, postoperative open-eye view after 2.5 months; (f) secondary division of the pedicle, postoperative closed-eye view after 2.5 months.

supported with an overlying skin graft, bridge flap (Cutler-Beard), temporal forehead flap (Fricke), semicircular rotational flap (Tenzel) and horizontal V-Y myotarsocutaneous advancement flap cannot reach the goals. Although the anterior lamella with placement of an overlying skin graft and bridge flap (Cutler-Beard) do have a good donor-recipient conjunctiva match, it has several limitations. These include prolonged occlusion of one eye, persistent lower eyelid instability, no lash restoration, no smooth eyelid margin, different color, thickness and character, risk of lid ectropion, and compromising lower eyelid integrity and stability.,4 The temporal forehead flap (Fricke) has the advantage of reconstruction of large anterior lamella defects; however, it is limited in that it requires posterior lamella coverage, requires removal of some original skin, the lack of eyelashes and eyebrows, which compromises lower eyelid integrity and stability, different color and thickness, no lash eyelid margin, and often needs a two-stage procedure. ${ }^{5,6}$ The semicircular rotational flap (Tenzel) also requires that the conjunctiva is undamaged; it has several limitations, such as no lash eyelid margin and the lack of eyelashes. It can be used to supplement reconstruction of the upper eyelid defect. ${ }^{7}$ Tarsoconjunctival substitutes by using an inferiorly based orbital septal flap that is reflected downwards, onto which the auricular cartilage is placed. The graft usually requires thinning, by removing the fatty 

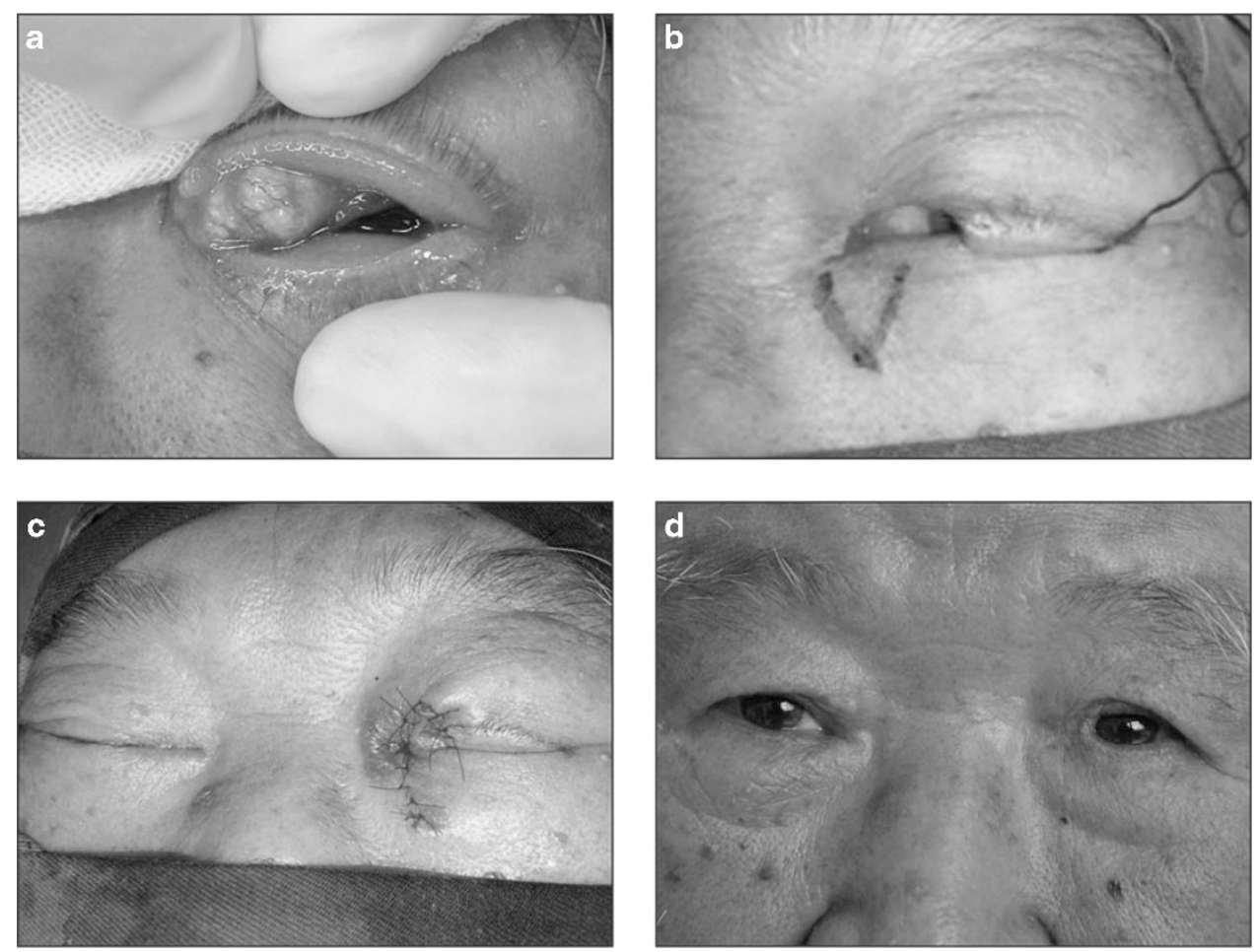

Figure 2 73-year-old male. (a) Preoperative view; (b) local resection creating a $1.6 \times 0.9 \mathrm{~cm}^{2}$ defect; (c) transfer of the flap to the upper eyelid defect; (d) postoperative view after 13 months.
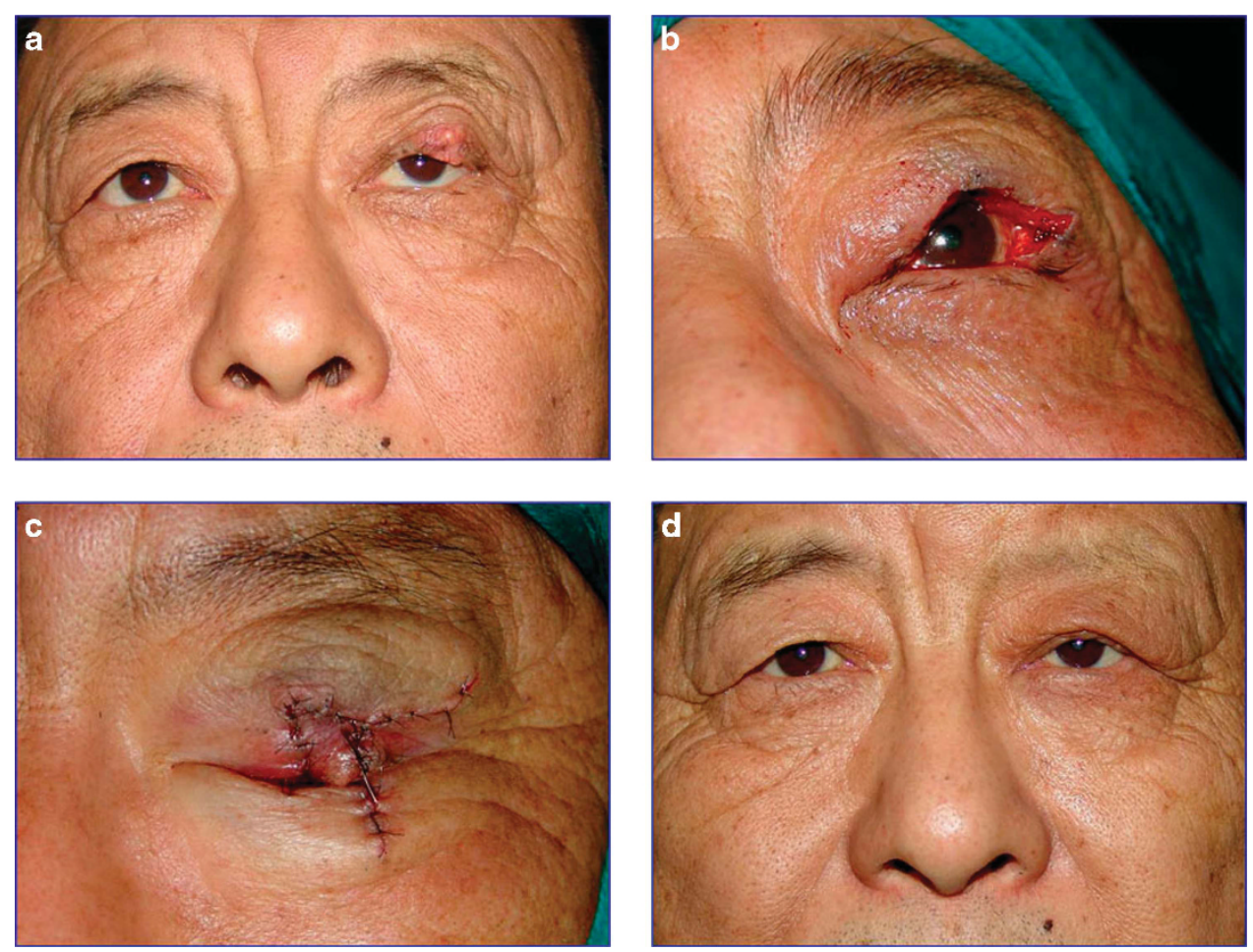

Figure 3 64-year-old male. (a) Preoperative view; (b) local resection creating a $2.2 \times 1.8 \mathrm{~cm}^{2}$ defect; (c) transfer of the flap to the upper eyelid defect; (d) postoperative view after 12 months. 
submucosa with scissors. Its limitations include secondary lesions, no symmetry of eyelid height and contour, and it may affect the function of the eyelid closure. $^{8-10}$ The horizontal V-Y myotarsocutaneous advancement flap has a better aesthetic result compared with full-thickness skin grafts for anterior lamellar reconstruction, but this method requires posterior lamella coverage. ${ }^{11}$ Its limitations are that there is no lash eyelid margin or eyelashes. It is difficult to use this means when anterior lamella defects involve over $30 \%$ of the eyelid.

In order to achieve the best reconstruction result, we followed the 'like-for-like' principle. The ipsilateral lower eyelid was the preferred source for such flaps and is the best option for full-thickness upper eyelid defects. While this flap is described in most plastic surgery textbooks, no further studies or clinical cases were published regarding the use of this particular flap in upper eyelid reconstruction. ${ }^{12-13}$ The lower eyelid provides an acceptable cosmetic appearance in relation to upper eyelid defects, with regards to symmetry of the eyelid, skin fold, color, thickness, character, contour, and eyelashes. Thus, the mucosa protects the underlying cornea, the tarsus offers stability to the eyelid, the orbicularis enables enable eyelid closure, the loose eyelid skin allows mobility, and the tight eyelid skin and lid margin prevent occlusion or irritation of the cornea by eyelid skin (Figure 1). Unfortunately, such flaps require secondary division of the pedicle. Alternatively, when advancing a full-thickness flap from the ipsilateral lower eyelid to repair a defect in the upper eyelid including the medial or lateral angle of the eye, a one-stage approach can be used (Figure 2). According to the actual need, medial or lateral cantholysis can be used in two-step procedures. It is important to keep in mind the close proximity of the lacrimal system to the medial canthal region for proper diagnosis and treatment. Care should be taken to avoid damaging the lacrimal gland and its secretory ductules. When the lacrimal system has been compromised following injury or cancer resection, it cannot be taken into account. The horizontal length of the pedicled lower lid full-thickness flap is less than that of the defect's width, so as to ensure an equal width between upper eyelid and lower eyelid after reconstruction. Functional and cosmetic results of pedicled lower lid-sharing flaps are highly acceptable to patients (Figure 3). Defects involving over two-thirds of the entire upper eyelid are difficult to repair by pedicled lower lid-sharing flaps, and require the recruitment of more distal tissue such as Cutler-Beard and the anterior lamella, with skin graft techniques.

\section{Summary}

What was known before

- Acute retinal necrosis (ARN) is a rare but visually devastating condition caused by human herpes viruses.

- However, no further studies or clinical cases were published.

\section{What this study adds}

- This study discusses on 13 clinical cases (including lateral cantholysis and Z flap, and medial cantholysis plasty in a one-step procedure).

\section{References}

1 Morley A, deSousa JL, Selva D, Malhotra R. Techniques of upper eyelid reconstruction. Surv Ophthalmol 2010; 55: 256-271.

2 Alghoul M, Pacella SJ, McClellan WT, Codner MA. Eyelid reconstruction. Plast Reconstr Surg 2013; 132: 288-302.

3 Fischer T, Noever G, Langer M, Kammer E. Experience in upper eyelid reconstruction with the Cutler-Beard technique. Ann Plast Surg 2001; 47: 338-342.

4 DiFrancesco LM, Codner MA, McCord CD. Upper eyelid reconstruction. Plast Reconstr Surg 2004; 114: 98-107.

5 Irvine $\mathrm{F}, \mathrm{McNab} \mathrm{AA}$. A technique for reconstruction of upper lid marginal defects. Br J Ophthalmol 2003; 87: 279-281.

6 deSousa JL, Malhotra R, Davis G. Sliding tarsal flap for reconstruction of large, shallow lower eyelid tarsal defects. Ophthal Plast Reconstr Surg 2007; 23: 46-48.

7 Codner MA, McCord CD, Mejia JD, Lalonde D. Upper and lower eyelid reconstruction. Plast Reconstr Surg 2010; 126: 231-245.

8 Wilcsek G, Leatherbarrow B, Halliwell M, Francis I. The 'RITE' use of the Fricke flap in periorbital reconstruction. Eye 2005; 19: 854-860.

9 Leibovitch I, Malhotra R, Selva D. Hard palate and free tarsal grafts as posterior lamella substitutes in upper lid surgery. Ophthalmology 2006; 113: 489-496.

10 Ito R, Fujiwara M, Nagasako R. Hard palate mucoperiosteal graft for posterior lamellar reconstruction of the upper eyelid: histologic rationale. J Craniofac Surg 2007; 18: 684-690.

11 Rosa J, Casal D, Moniz P. Upper eyelid reconstruction with a horizontal V-Y myotarsocutaneous advancement flap. J Plast Reconstr Aesthet Surg 2010; 63: 2013-2017.

12 Mustarde JC. Reconstruction of the eyelids. Ann Plast Surg 1983; 11: 149-169.

13 Mustarde JC. New horizons in eyelid reconstruction. Int Ophthalmol Clin 1989; 29: 237-246. 